\title{
Research of HID (Human Interface Device) 3-Dimensional Motion Recognition Device
}

\author{
Min-Seok $\mathrm{Jie}^{1}$, Seung-Hun $\mathrm{Kim}^{2}$ and Won-Hyuck $\mathrm{Choi}^{3 *}$ \\ ${ }_{1,3}$ Department of Avionics Engineering Hanseo University \\ 236-49, Gomseom-ro, Nam-myeon, Taean-gun, 32158 Chungcheongnam-do, \\ Republic of Korea \\ ${ }^{2}$ Department of Aeronautical Systems Engineering Hanseo University \\ 236-49, Gomseom-ro, Nam-myeon, Taean-gun, 32158 Chungcheongnam-do, \\ Republic of Korea \\ $\left\{{ }^{1}\right.$ choiwh, ${ }^{3}$ jiems $\} @$ hanseo.ac.kr, ${ }^{2}$ Kimsh014@gmail.com
}

\begin{abstract}
In this paper, we developed a wireless transceiver based 3D motion recognition device for HMD (Head Mounted Display). This unit uses a gyro sensor and acceleration sensor. A device that recognizes the user's head and body parts in 3D motion. You can recognize it by attaching it to an object. Human interface devices (HID) systems also connect directly to the user. It utilizes the user's movement obtained from the device. The Kalman filter is used to increase the accuracy of the data. It connects to UAV and provides more accurate information and freedom of space. It can be applied to all areas of the remote control.
\end{abstract}

Keywords: Motion Sensor, Motion Recognition, Remote Control, HID, HMD

\section{Introduction}

The theme of modern technology is convergence. Convergence is a business that increases value by providing new products or services by utilizing existing ones or by combining two or more technologies. Because it can produce high value-added products according to the characteristics of industry [1].

A long time ago, convergence was attempted based on ICT technology in aeronautics, which requires cutting-edge technology. This applies to aircraft and related aircraft. The equipment provides high reliability for the convenience and safety of pilots, aircraft operation, and safety. In recent years, the most active area of aviation ICT technology has been merged with avionics. Industry characteristics in avionics field are possible. Create high added value. Also, product utilization is high. The spin-off is actively proceeding to aviation ICT convergence technology, avionics, automobile and mobile platform. HMD and augmented reality technology representing hardware and software [2].

In this paper, we developed a motion recognition system for HMD camera gimbal control of the unmanned aerial vehicle. An important part of motion recognition devices is data. This is because the camera gimbals must move precisely according to the movement of the user's head. The Kalman filter algorithm is used for data stabilization. So, by designing HID-based wireless communication protocols through motion, remote operations can be controlled and performed easily with smart devices.

Received (December 17, 2017), Review Result (March 20, 2018), Accepted (March 26, 2018) 


\section{Related Studies}

\subsection{Motion Recognition}

First, gyro sensors and motion sensors must detect user movement.

A gyro sensor that measures the Earth's gravity is used as the primary sensor to identify motion.

The gyro sensor is a sensor that can measure the angular acceleration of a moving object. By using the gyro sensor, it is possible to recognize the $3 \mathrm{D}$ motion by accurately measuring the movement coordinate value of the user.

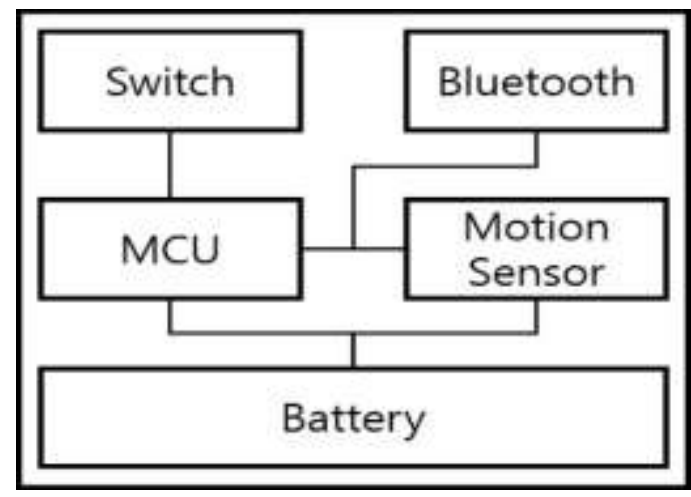

Figure 1. Block Diagram

Figure 1 is basically supplied with $5 \mathrm{~V}$ power through the battery, and supplies power to the motion sensor, MCU (Micro Controller Uint) and Bluetooth.

The position data of the motion sensor is transmitted to the MCU.

The MCU will organize the received data and stabilize the data. The collected data is transmitted using Bluetooth wireless communication.

The device operates at the same time as the power supply is applied. The switch in the figure can perform a device reset through the reset button when the device does not operate as a reset button or an error occurs.

\subsubsection{Gyro Sensor}

The gyro sensor is a sensor that measures the input angular velocity in each environment. The gyro sensor has design parameters such as rotating axis, inertia moments, elastic modulus, load factor, shaft inertia, etc. This determines the gyro stability and performance of the gyro, depending on the environment where the gyro is used. The gyro sensor can detect rotational motion and direction changes and increase movement. Vibration gyro sensors can detect the angular velocity caused by vibration elements of vibrating corium. This movement creates a potential difference where the angular velocity is sensed. The angular velocity is converted to an electrical signal output.

A gyrostat consists of a massive flywheel concealed in a solid casing. It's behavior on a table, or with various modes of suspension or support, serves to illustrate the curious reversal of the ordinary laws of static equilibrium due to the gyrostatic behavior of the interior invisible flywheel when rotated rapidly. The first gyrostat was designed by Lord Kelvin to illustrate the more complicated state of motion of a spinning body when free to wander about on a horizontal plane, like a top spun on the pavement, or a bicycle on the road. Kelvin also made use of gyrostats to develop mechanical theories of the elasticity of matter and of the ether. In modern continuum mechanics there is a variety of these models, based on ideas of Lord Kelvin. They represent a specific type of Cosserat theories (suggested for the first time by Eugène Cosserat and François Cosserat), which can be used for description of artificially made smart materials as well as of other complex media. One 
of them, so-called Kelvin's medium, has the same equations as magnetic insulators near the state of magnetic saturation in the approximation of quasi magnetostatics [34].

$$
\mathrm{F}=\operatorname{mr}(\mathrm{w}+\Omega)^{2}
$$

In this equation, $v=r w$ and $m$ are the rotational velocity with respect to the plate. The first term of equation (1) is the centrifugal force measured at the coordinates based on the flat plate. The second term is the force of the vector product of the rotation $\mathrm{w}$ of $\mathrm{m}$ and the rotation $\Omega$ of the plate. It is a force perpendicular to $\mathrm{v}$ and $\Omega$, and this is the force of Coriolis.

\subsection{HID(Human Interface Device)Based Wireless Communication}

The HID is a human interface device and is a means of charging the user interface of the peripheral devices around the computer. This means that it receives inputs from almost human beings and sometimes includes multiple output functions. A developed device is a contact-based user interface that attaches a sensor or device to a user's body region. Leverage user-motion information from sensors and devices connected. The advantage of the contact method is that you can leverage the $3 \mathrm{D}$ information of your user behavior to gain accurate information about the user's behavior in interacting with 3D virtual objects.

BLE is an advanced communications protocol using a small, low-power digital radio based on IEEE 802.15.4-2003. IEEE 802.15.4-2003 is the standard for short-range personal wireless networks, such as lamps, electronic meters, and consumer electronics that use short-range radio frequencies. BLE is primarily used in RF applications that require low data rates, low battery consumption, and network security. BLE is currently used for embedded sensors, industrial control, medical data collection, building automation, fire and theft, and home automation.

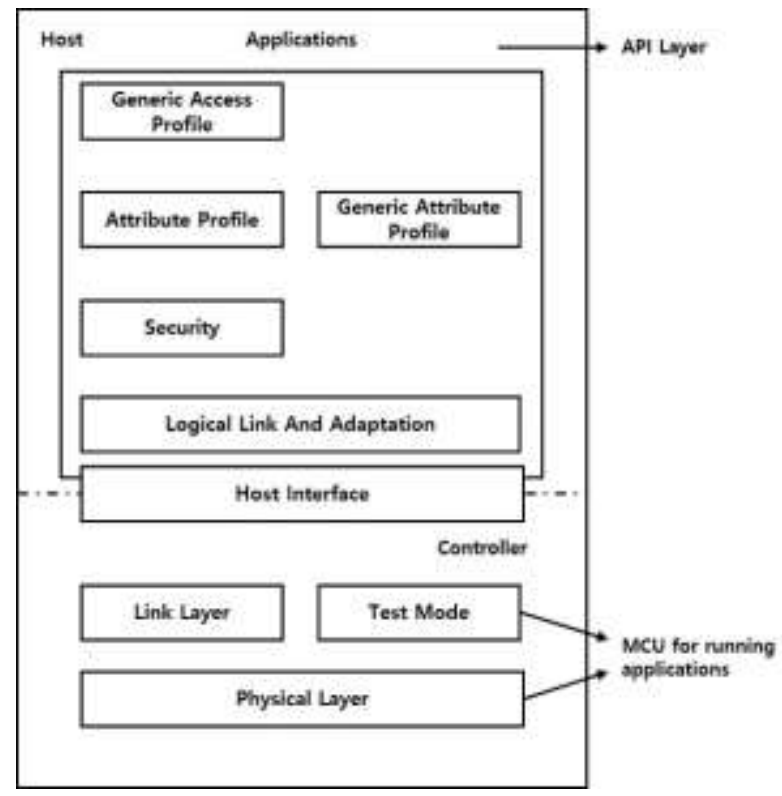

Figure 2. BLE Stack Diagram

\subsection{Gimbal System}

The stabilization platform is based on a two-axis gimbal structure with a camera, pointing device, and inertia sensor. Both the camera and pointer can be controlled individually, and the pointer's upward and downward movement is synchronized with that of the camera. 


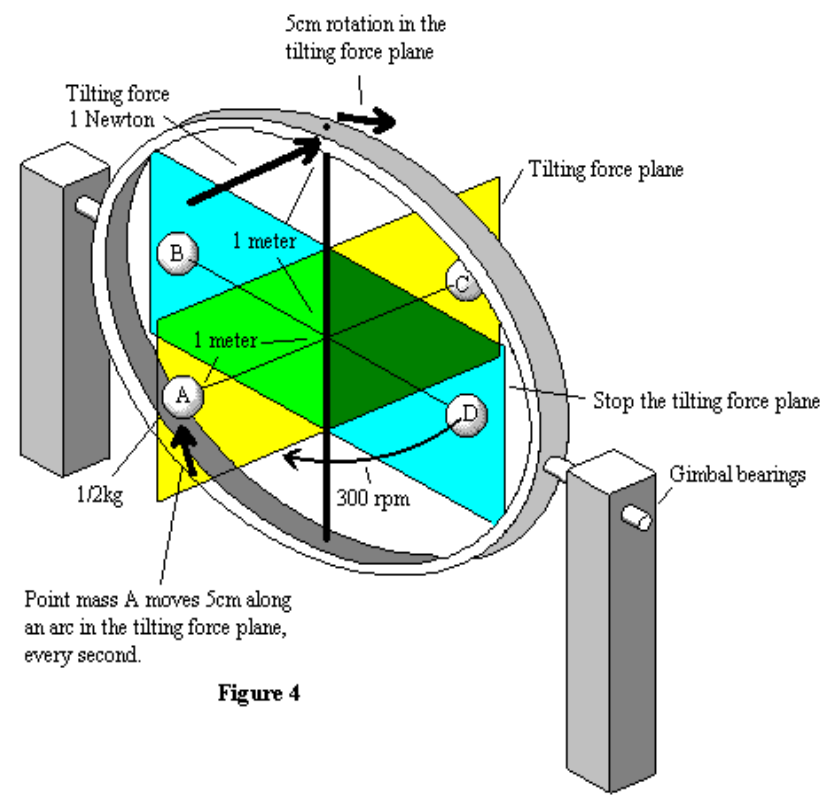

Figure 3. Structure of the Gyro

Rounding is a two-piece system that is implemented through a gearbox connected between the motor and the rod, where each motor is attached with a resolver and encoder to transmit position signals. For stability control, the platform is also equipped with a strap down inertia sensor (Three-axis gyro and accelerometer). The stabilization platform is divided into non-sanitarized mode used for manual operation by the joystick and stability mode to compensate for changes in the attitude of the mounted objects (ships) according to the terrain and environmental conditions. Non-valibration mode is controlled using Lizzover's feedback signal, and the stabilization mode uses the speed feedback signal using the inertia sensor.

Stability mode is such that the steering line follows the desired target point when an outer line is present, where a typical inertially-modulated sensor's inertia platform (ISP) is used to measure the outer configuration of a platform. Therefore, it is making compensation using gyro and Rizzolber's signals. However, when using the gyro alone, it is difficult to compensate for pitch motion within the inertia coordinate system that occurs when the boat is tilted, and to compensate for foreign limit due to sensor error and drift. The accumulation of these errors eventually leads to the deflection of the pointer device. The principle of gimbal operation is identical to that of the main controller, ATMega 2560, which has the advantage of having many input and output ports and fast handling speed. Due to the large number of communication ports, Bluetooth communication ports and IMU communication ports can proceed simultaneously, making them the main controllers.

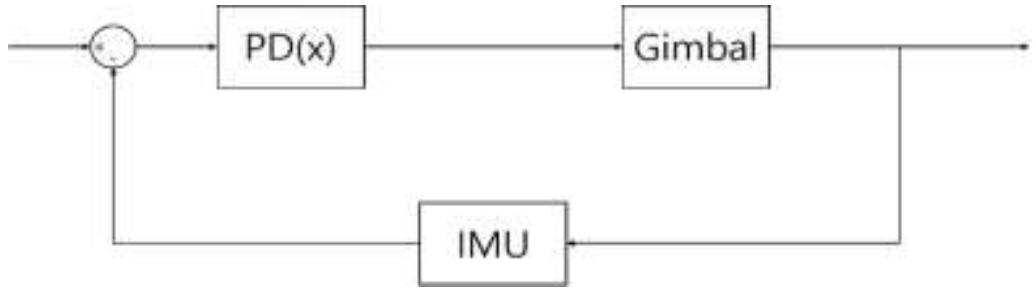

Figure 4. Control Block Diagram of Gimbal System

Although there are ways to add a position control loop to make precise pitching rewards without significantly changing the existing stability control structure, two major issues must be considered when controlling positions. First, there are problems where the feedback 
signals from the speed loop may interfere with the position control, offset the position control, and difficulty in selecting the target values to be used for the position control. For target values, information from vision sensors and cameras can determine the target Encoder value, but for platforms to be used in this study, only the inertia sensor value should be selected without such additional information.

To solve these problems, this study has designed a reference angle for precision pitch compensation. This reference angle saves the current posture (pitch) and the Encorder angle, and uses the saved position angle after the start of the control to produce the target values required for position control.

Figure 4 shows the PID control for the basic attitude measurement and provides a stable data measurement without the need for any special PID control.

\section{System Composition}

The system proposed in this paper is a system that remotely controls an object using motion sensors using a gyro sensor and an acceleration sensor. The system configuration is shown in Figure 5. The system is divided into four parts, which are mounted at the position for the user's head and operation, and are used to measure the position and position of the IMU sensor. The IMU sensor is a combination of a gyro sensor and an acceleration sensor, not a specific sensor, and is developed based on the MPU9250 sensor. In addition, the yaw value is stabilized using a geomagnetic sensor. The Yaw value is a rotation value about the $\mathrm{Z}$ axis, and is used as data to be compensated without using a direct value. Since the data of the geomagnetic sensor is unstable by the peripheral devices and oneself, it is judged that it is impossible to use it directly, and the data is processed as compensation data.

The measured data was transferred to the MCU, the data for each location was integrated, and the data was collected based on the Kalman Filter.

The stylized data is transferred to the gimbal device using the Bluetooth wireless communication, and the Servo Motor and the BLDC (Brushless DC Motors) are operated to move the camera directly in the direction to see the user.

Measured data is transferred to MCU, integrated data for each location, and cleaned up data based on Kalman Filter.

It forwards the formatted data to the Gimbal Device using Bluetooth's wireless communication, and then moves the Servo Motor and Brushless DC Motors (BLDC) to determine the location to which the user will be directed.

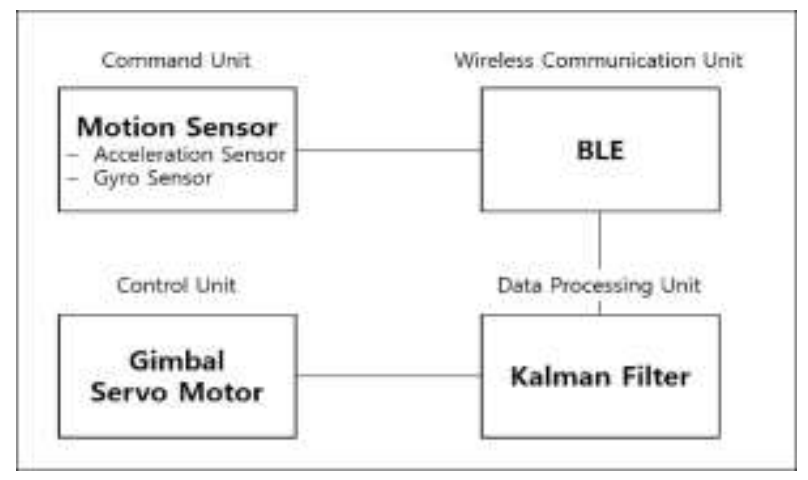

Figure 5. System Diagram

To control the user's head movements and remotely control, the system is driven in the sequence shown. The transmitter is in the user's head and calculates the angular value from the motion sensor and the receiver is the sensor value. A small board was designed for experiments. The BLE communication module enables communication between the 
transmitter and receiver between the transmitter and receiver, the SPI communications between boards and BLE, and I2C communication between the boards and motion sensors.

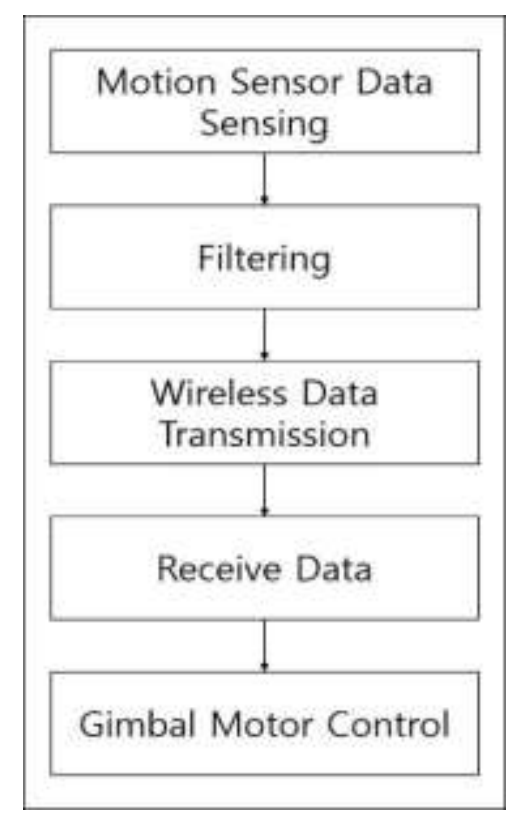

Figure 6. System Flowchart

\subsection{Sample Board}

The following is a sample board. The sample board injects the PCB substrate based on the design of the $\mathrm{H} / \mathrm{W}$ and consists of the IMU, the communication part, the LED part and the power part.

This configuration includes the MPU-9250, a 9-axis sensor for calculating the processor and acceleration and angular velocity. Since the MPU-9250 sensor includes a gyro sensor and an acceleration sensor, the PCB is selected for fabrication and miniaturization.

The battery must be selected to supply power to the device. The size of the battery is limited in consideration of mobility and mountability, and a coin type mercury battery is adopted.

We used the ATMega 2560, which we had done for the previous test, but it was made for a small-sized processor for miniaturization and mobility [nRF51822]. In the case of this processor, Bluetooth wireless communication was included, so it did not design the Bluetooth module separately and played a big role in reducing the size. The size of this sample board is $4 \times 4 \mathrm{~cm}$ and it is made suitable for miniaturization.

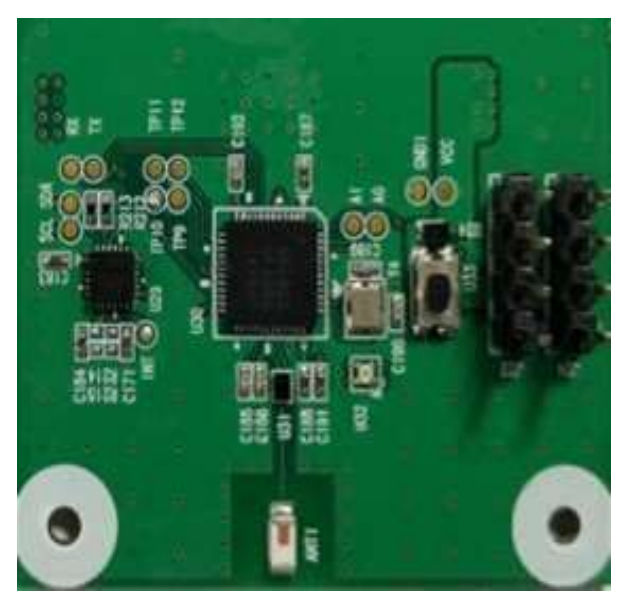

Figure 7. System Flowchart 


\subsection{Simulation}

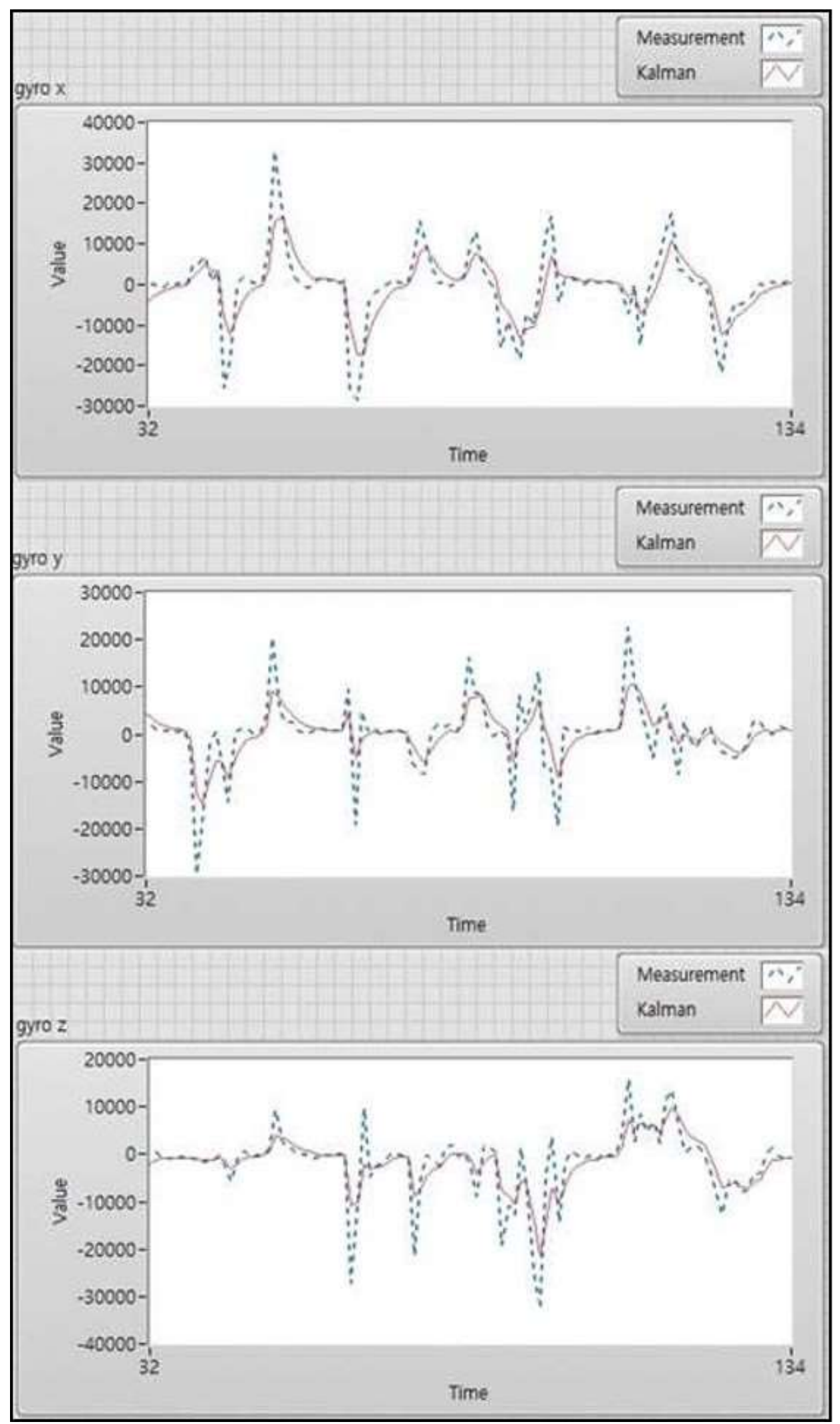

Figure 8. Gyro Sensor Graph 


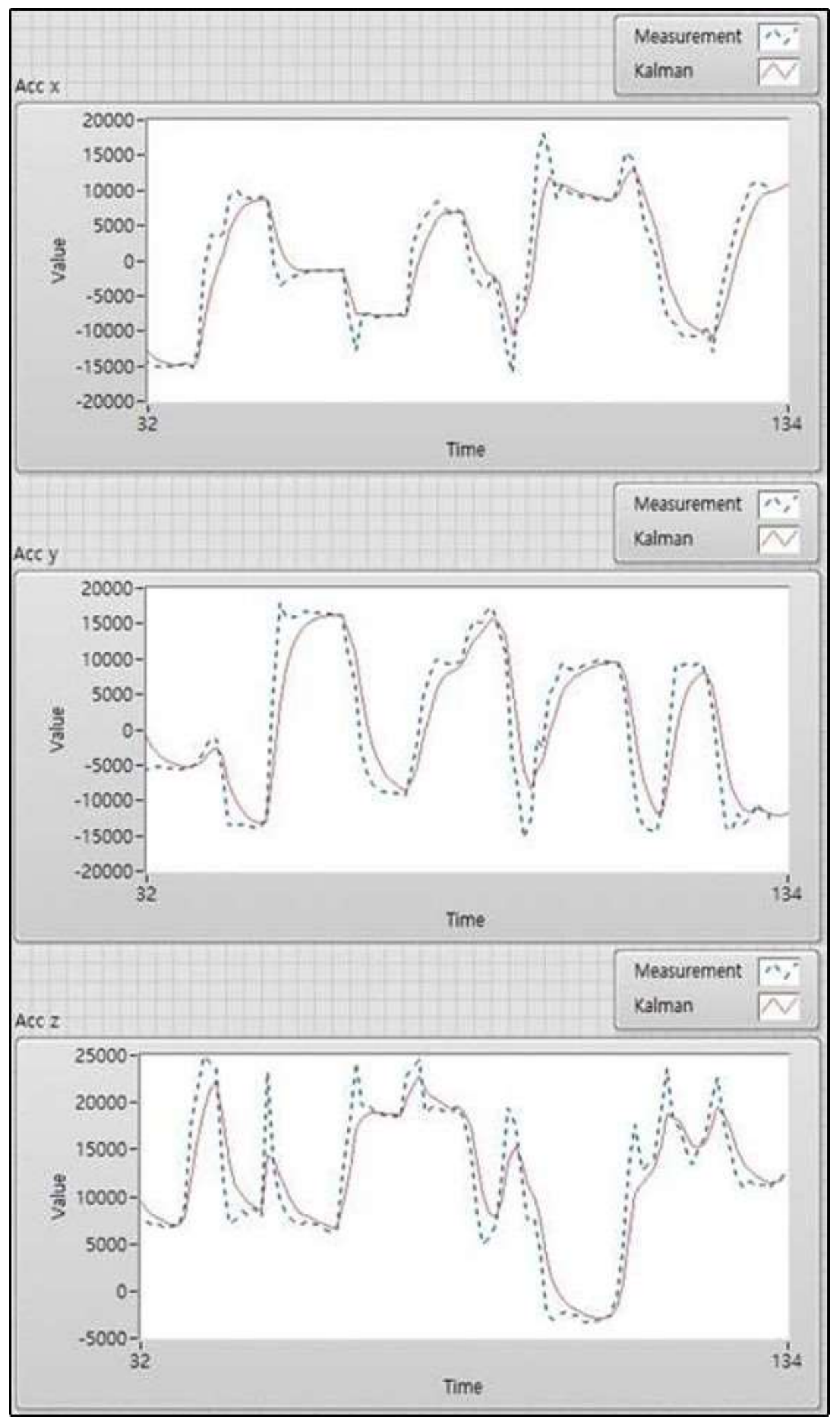

Figure 9. Accelerometer Sensor Graph 


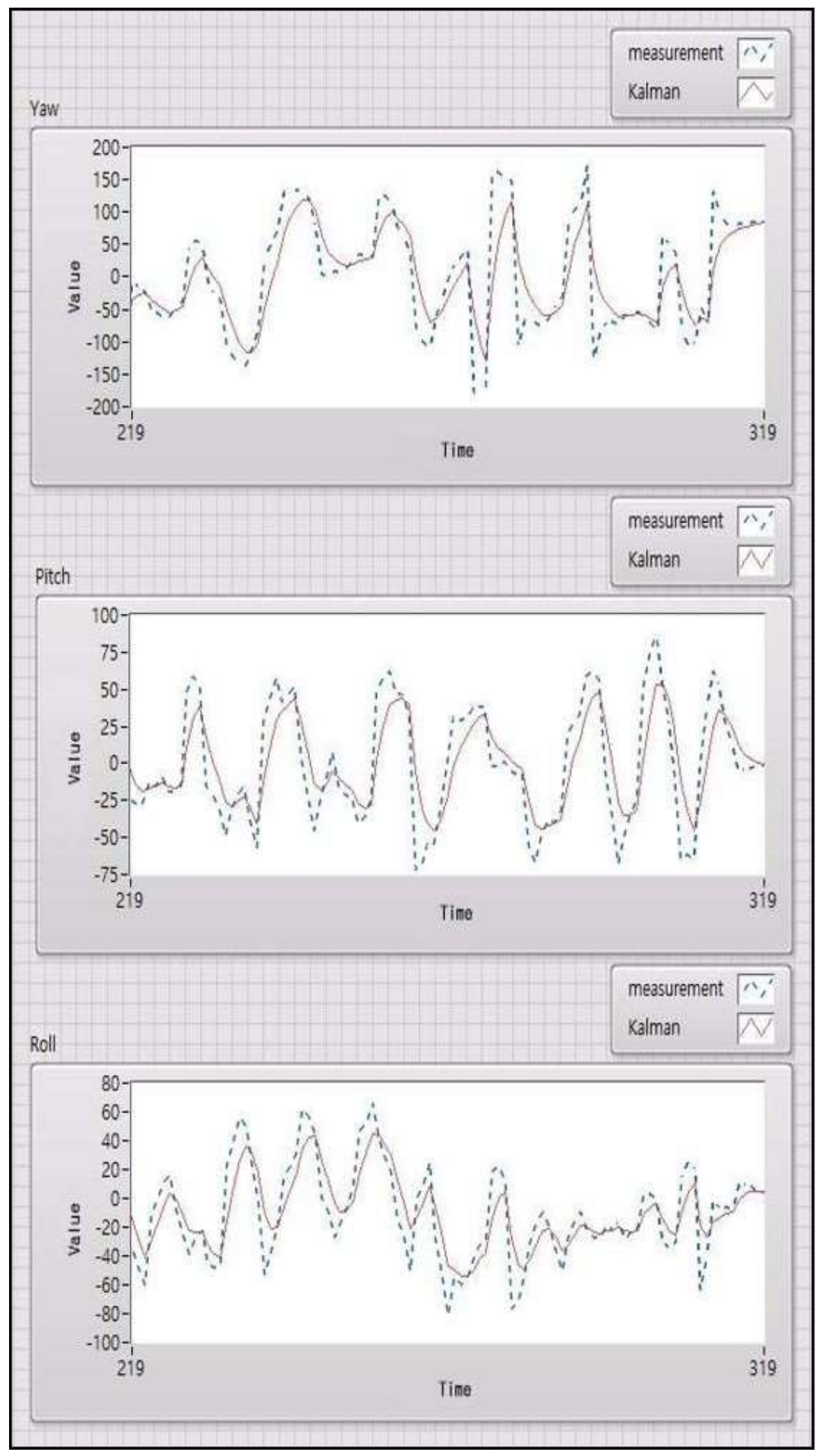

Figure 10. Roll, Pitch, Yaw Data Graph

In this paper, we evaluate the usability of the developed device by remote experiment. By attaching the motion recognition device to the user's head and collecting data remotely, the data is displayed in LabVIEW graph in real time. Moving the user's head in various 
directions confirmed the changing data. In addition, we confirmed a more stable data output using the Kalman Filter algorithm [10].

\section{Conclusion}

In this paper, we developed a motion recognition device for camera gimbal. By developing a motion recognition device, it can control the object remotely, such as a camera gimbal. That is, the gimbal camera can control the camera in the same way as the head movement of the user. Therefore, this device is more intuitive and convenient recognition. And As shown in Figure 10 Figure 11 Figure 12, the Kalman filter algorithm enables more stable motion. and This device, as well as smart control of the device is applicable to any field that requires the remote control.

We experimented with LabVIEW to test the stability of the developed device. The developed device and the computer were connected wirelessly to confirm the data in real time. And confirmed stable data. The motion recognition check the user's midair motion, and designing the HID-based wireless communication protocol, it is possible to control and easy remote work with the smart device.

\section{Acknowledgment}

This work was supported by 2017 research grant from Hanseo University.

\section{References}

[1] K. R. Lee and J. R. Hwang, “A Study on Innovation System with Multi-technology Fusion”, Science and Technology Policy Institute., Korea, (2005).

[2] H. J. Park and T. H. Han, "Augmented Reality-based E-Learning Technology Trends", Review of Korean Society for Internet Information., vol. 10, no. 2, (2009), pp. 12-22.

[3] X. Liu and J. Li, "A Smart Energy-efficiency Deployment Scheme for Lifetime Enhancement Wireless Sensor Networks", Journal of The Smart Computing Review., vol. 5, no. 6, (2015), pp. 591-601.

[4] I. A. Khan and F. Ahmad, "Application-based Classification and Comparison of Secure Routing Protocols in Wireless Sensor Networks - A Survey”, Journal of The Smart Computing Review, vol. 5, no. 3, (2015), pp. 209-223.

[5] D. P. Hong and W. T. Woo, "Recent Research Trend of Gesture-based User Interfaces", Telecommunication Review, vol. 18, no. 3, (1981), pp. 403-413.

[6] D. Li and J. Lee, "Minimum Gradient Forwarding for Wireless Sensor Networks", Journal of The Smart Computing Review., vol. 4, no. 5, (2014), pp. 418-425.

[7] L. C. Ko and J. S. Lee, "ZigBee Security for Residential Sensor Networks", Journal of The Smart Computing Review., vol. 1, no. 2, (2011), pp. 95-103.

[8] M.G. Song, "Development of Gimbal System for Aviation Photograph using Smartphone", Korea Society of Precision Engineering. (2014), pp. 294.

[9] A. Mehmood and H. Song, "Smart Energy Efficient Hierarchical Data Gathering Protocols for Wireless Sensor Networks", Journal of The Smart Computing Review., vol. 5, no. 5, (2015), pp. 425-462.

[10] H.O. Jang Editor, "Understanding the LabVIEW Graphics Program", ADC System, Korea, (1998).

\section{Authors}

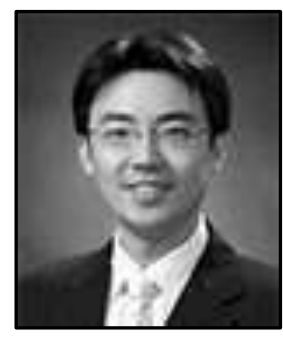

Min-Seok Jie, he graduated from the Ph.d degree in the department of avionics Engineering from Korea Aerospace University in 2006. he is Hanseo University Avionics Engineering Professor in to the present in 2008. 


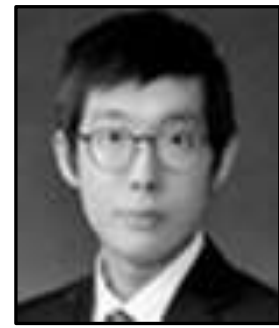

Seung-Hun Kim, he is a master's degree in Aeronautical System Engineering, Hanseo University. He graduated from the Department of Aeronautical and Mechanical Engineering, Hanseo University. He is studying Kalman filters, embedded systems, and smart network communications.

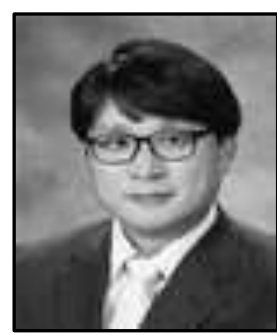

Won-Hyuck Choi (Corresponding author), He received the Ph.D. degree in avionics from Korea Aerospace University, Korea. He was a Professor of smart network at Doowon Technical University College, Korea. urrently, he is Professor of avionics at Hanseo University, Korea, where he has been since 2014. His present research interests include embedded system, home network. 
International Journal of Advanced Science and Technology Vol.117 (2018) 